\title{
Evolution of the dependence of residual lifetimes
}

\author{
Fabrizio Durante ${ }^{1}$ and Rachele Foschi ${ }^{2}$
}

\begin{abstract}
We investigate the dependence properties of a vector of residual lifetimes by means of the copula associated with the conditional distribution function. In particular, the evolution of positive dependence properties (like quadrant dependence and total positivity) are analyzed and expressions for the evolution of measures of association are given.
\end{abstract}

\section{Introduction}

In the present note, we are interested in multivariate stochastic models related to a system composed by several components, whose behaviour can be represented by a random vector $\mathbf{X}=\left(X_{1}, X_{2}, \ldots, X_{d}\right)$ defined on a suitable probability space and taking values in $\mathbb{R}_{+}^{d}$. Specifically, each $X_{i}$ is a continuous and positive random variable having the meaning of lifetime. For instance, in reliability theory, $X_{i}$ 's represent the lifetimes of certain disposals working in the same system; in credit risk, $X_{i}$ 's may represent the times-to-default of some companies. Regardless of their specific interpretation, it has been long recognized that the behaviour of $\mathbf{X}$ depends on both the individual behaviour of each component and the dependence structure of $\mathbf{X}$ as interpreted by its copula $C$ (see [10] and the references therein).

Here we are interested in the evolution of the dependence structure when one knows that all the components of $\mathbf{X}$ have survived up to time $\tau>0$. For such a situation, the following facts can be revealed:

Free University of Bozen-Bolzano, School of Economics and Management, Bolzano (Italy), e-mail: fabrizio.durante@unibz.it . IMT Advanced Studies, Lucca (Italy), e-mail: rachele.foschi@imtlucca.it 
- the evolution of the dependence has "no jumps", in the sense that it evolves smoothly and does not admit drastic changes (as it would be in presence of exogenous shocks);

- the evolution of the dependence is stable with respect to misspecification, in the sense that "small" errors in selecting the dependence structure at time $\tau=0$ do not amplify;

- some "weak" positive dependence among the components of $\mathbf{X}$ may disappear when $\tau$ increases. Contrarily, a "stronger" positive dependence is preserved at any time $\tau$.

In the following, we clarify how these facts can be described rigorously in terms of copulas and present some additional results, especially concerning association measures, and relevant examples.

\section{Copulas of residual lifetimes}

For sake of simplicity, we treat only the case $d=2$ (most of the considerations can be easily extended to the general case). Thus, let us consider a pair $\left(X_{1}, X_{2}\right)$ of lifetimes whose copula is given by $C$. We denote by $\mathscr{C}$ the class of bivariate copulas.

As known (see [4]), the dependence properties of the family of distribution functions $\left(F_{\tau}\right)_{\tau \geq 0}$ of

$$
\left[X_{1}-\tau, X_{2}-\tau \mid X_{1}>\tau, X_{2}>\tau\right]
$$

can be described by means of a suitable copula process $\left(C_{\tau}\right)_{\tau>0}$. Moreover, it is convenient to reparametrize the latter copula process in terms of a parameter $t \in(0,1]$, obtaining the process $\left(C_{t}\right)_{t \in(0,1]}$. In other words, $C_{1}$ represents the dependence structure of $F_{\tau}$ when $\tau=0$ and, as $t$ tends to $0, C_{t}$ represents the limiting dependence structure of $F_{\tau}$ as $\tau$ tends to $\infty$.

The following result allows us to derive some analytical properties of the copula process $\left(C_{t}\right)_{t \in(0,1]}$.

Proposition 1 ([4]). For every $t \in(0,1], C_{t}$ is completely described by the restriction of the copula $C$ to $[0, t]^{2}$. Specifically, one has

$$
C_{t}(u, v)=\frac{C\left(h_{t}^{-1}\left(u h_{t}(t)\right), k_{t}^{-1}\left(v k_{t}(t)\right)\right)}{C(t, t)},
$$

where $h_{t}(u)=C(u, t)$ and $k_{t}(u)=C(t, u)$ for all $t \in[0,1]$.

Formally, the transformation (1) can be described in terms of the following mapping:

$$
\Psi:(0,1] \times \mathscr{C} \rightarrow \mathscr{C}, \quad \Psi(t, C)=C_{t} .
$$

Such a $\Psi$ has the following features: 
- Let $C \in \mathscr{C}$. The mapping $\Psi(\cdot, C):(0,1] \rightarrow \mathscr{C}, t \mapsto C_{t}$, is continuous, i.e., $C_{t}$ converges uniformly to $C_{t_{0}}$ when $t$ tends to $t_{0}$. Roughly speaking, the evolution of the dependence has no jumps (see [4]).

- Let $t \in(0,1]$. The mapping $\Psi(t, \cdot): \mathscr{C} \rightarrow \mathscr{C}$ is continuous with respect to the $L^{\infty}$-norm. In other words, if the copulas $C$ and $C^{\prime}$ are sufficiently close each other (with respect to a suitable norm), then, for any $t, C_{t}$ and $C_{t}^{\prime}$ are sufficiently close each other (see [1]). Roughly speaking, the evolution of the dependence is stable with respect to misspecification of $C_{1}$.

Moreover, notice that $\Psi$ can be interpreted as the action of a suitable semigroup $((0,1], *)$ on $\mathscr{C}$. In particular, for all $t, s \in(0,1]$, we have $\left(C_{t}\right)_{s}=$ $\Psi\left(s, C_{t}\right)=C_{t * s}($ see $[8,9])$.

It can be easily seen that the limit of $C_{t}$, as $t$ tends to 0 , may not exists. To this end, it is enough to consider a special kind of ordinal sum of copulas or a copula with fractal support (see, for instance, [1, Remark 3.3]). However, when such a limit exists, it follows that the limiting copula is invariant under the transformation defined in Eq. (1). For example, the independence copula $\Pi(u, v)=u v$ and the comonotone copula $M(u, v)=\min (u, v)$ are invariant; moreover copulas belonging to the Clayton family of copulas $\left\{C_{\theta}^{C l}\right\}$,

$$
C_{\theta}^{C l}(u, v)=\left(\max \left(0, u^{-\theta}+v^{-\theta}-1\right)\right)^{-1 / \theta}, \quad \theta \geq-1, \theta \neq 0,
$$

are invariant (for more details, see $[5,6]$ ).

\section{Dependence of residual lifetimes}

Now, suppose that $C \in \mathscr{C}$ satisfies some positive dependence property. Our aim is to investigate whether the positive dependence is preserved by the process $\left(C_{t}\right)_{t \in(0,1]}$. First we introduce some definitions (see, e.g., [11]).

Definition 1. Let $C \in \mathscr{C}$.

- $C$ is PQD (positive quadrant dependent) if and only if $C(u, v) \geq u v$ for all $u, v \in[0,1]$.

- $C$ is TP2 (totally positive of order 2 ) if and only if for all $u, u^{\prime}, v, v^{\prime}$ in $[0,1], u \leq u^{\prime}, v \leq v^{\prime}$

$$
C(u, v) C\left(u^{\prime}, v^{\prime}\right) \geq C\left(u, v^{\prime}\right) C\left(u^{\prime}, v\right) .
$$

- $C$ is PLR (positively likelihood ratio dependent) if and only if it is absolutely continuous and its density satisfies (3).

Notice that PLR implies TP2; moreover, if $C$ is TP2, then $C$ is PQD. The following result also holds. 
Proposition 2 ([4]). Let $C \in \mathscr{C}$.

- If $C$ is TP2, then $C_{t}$ is TP2 for all $t \in(0,1]$.

- If $C$ is PLR, then $C_{t}$ is PLR for all $t \in(0,1]$.

If $C$ is PQD, instead, $C_{t}$ may not be PQD for some $t$ (see e.g. [4, Example 10]). In order to guarantee that positive quadrant dependence of $C$ is preserved by any $C_{t}$, we need some stronger conditions, as specified in the following result.

Proposition 3 ([4]). Let $C \in \mathscr{C}$. Then $C_{t}$ is $P Q D$ for all $t \in \Lambda \subseteq(0,1]$ if and only if, for all $u, v, t \in \Lambda, u, v \leq t$,

$$
C(u, v) C(t, t) \geq C(u, t) C(t, v) .
$$

In particular, $C$ is said to be hyper- $P Q D$ if $C$ satisfies (4) for $\Lambda=(0,1]$.

Another way to look at the dependence evolution of the process $\left(C_{t}\right)_{t \in(0,1]}$ consists in introducing a suitable way to compare the copulas at different times. To this end, we consider the following definitions.

Definition 2. Let $C_{1}, C_{2} \in \mathscr{C} . C_{1}$ is smaller than $C_{2}$ in the PQD order (written $C_{1} \preceq_{P Q D} C_{2}$ ) if $C_{1}(u, v) \leq C_{2}(u, v)$ for all $u, v \in[0,1]$.

Definition 3. Let $C \in \mathscr{C}$. Then $\left(C_{t}\right)_{t \in(0,1]}$ is increasing (in the PQD order) if $C_{t^{\prime}} \preceq_{P Q D} C_{t^{\prime \prime}}$ for any $t^{\prime}<t^{\prime \prime}$.

The following example shows that, regardless of the specific positive dependence of $C \in \mathscr{C}$, the dependence may evolve in different ways.

Example 1. Given a continuous and increasing function $f:[0,1] \rightarrow[0,1]$ such that $f(1)=1$ and $\frac{f(t)}{t}$ is decreasing on $(0,1]$, consider the copula

$$
C(u, v)=\min (u, v) f(\max (u, v))
$$

(see $[3,7]$ for more details about this construction). Such a copula $C$ is TP2 (see [3]). Therefore, as follows by condition (4), $C$ is hyper-PQD. Furthermore, by Proposition 2,

$$
C_{t}(u, v)=\min (u, v) \frac{f(t \max (u, v))}{f(t)}
$$

is TP2 for any $t \in(0,1]$. The evolution of the strength of the dependence, instead, is influenced by the choice of $f$. In particular, the following cases can be considered.

- If $f(t)=t^{\alpha}, \alpha \in[0,1]$, then $C$ is a Cuadras-Augé copula (see [2]) and $C_{t}=C$ for every $t \in(0,1]$ (see also [1, Example 4.1]).

- If $f(t)=\alpha t+(1-\alpha), \alpha \in[0,1]$, then $C$ is a Fréchet copula and, for all $t_{1}, t_{2} \in(0,1], t_{1} \leq t_{2}$, we have $C_{t_{1}} \succeq_{P Q D} C_{t_{2}}$. In particular, $\lim _{t \rightarrow 0^{+}} C_{t}(u, v)=\min (u, v)$. 
- If $f(t)=\min (\alpha t, 1), \alpha \geq 1$, then $C$ is an ordinal sum of the copulas $\Pi(u, v)=u v$ and $M(u, v)=\min (u, v)$ with respect to the partition $([0,1 / \alpha],[1 / \alpha, 1])$. Thus, for all $t_{1}, t_{2} \in(0,1], t_{1} \leq t_{2}$, we have $C_{t_{1}} \preceq_{P Q D} C_{t_{2}}$. In particular, $\lim _{t \rightarrow 0^{+}} C_{t}(u, v)=u v$.

Thus, depending on $f$, the mapping $t \mapsto C_{t}$ may be constant, increasing or decreasing in the PQD order.

\section{Measures of association of residual lifetimes}

The analysis of the dependence properties of $C_{t}$ can be sometimes complicated because of technical difficulties in computing Eq. (1). In such a case, it could be convenient to consider some suitable association measures that are related to copulas.

Here, we concentrate on the most widespread Kendall's tau and Spearman's rho, which measure the concordance between two random variables. As known (see e.g. [12]), for every copula $C$, they are given by:

$$
\begin{aligned}
& \tau_{K}(C)=4 \int_{[0,1]^{2}} C(u, v) d C(u, v)-1 \\
& \rho_{S}(C)=12 \int_{[0,1]^{2}}(C(u, v)-u v) d u d v .
\end{aligned}
$$

To compute such measures for $C_{t}$, we assume here that $C$ is absolutely continuous and, hence, $C_{t}$ is absolutely continuous for all $t \in(0,1]$ (see $[4$, Proposition 17]).

Proposition 4. Let $C$ be an absolutely continuous copulas. For every $t \in$ $(0,1]$, one has

- $\tau_{K}\left(C_{t}\right)=\frac{4}{C(t, t)^{2}} \int_{[0, t]^{2}} C(x, y) \partial_{12}^{2} C(x, y) d x d y-1$;

- $\rho_{S}\left(C_{t}\right)=\frac{12}{C(t, t)^{4}} \int_{[0, t]^{2}}^{(C, t]}(C(x, y) C(t, t)-C(x, t) C(t, y)) \partial_{1} C(x, t) \partial_{2} C(t, y) d x d y$.

Proof. By the formula for calculating Kendall's $\tau$, one has

$$
\tau_{K}\left(C_{t}\right)=4 \int_{[0,1]^{2}} C_{t}(u, v) \partial_{12}^{2} C_{t}(u, v) d u d v-1 .
$$

Therefore, by the change of variable $x=h_{t}^{-1}\left(u h_{t}(t)\right)$ and $y=k_{t}^{-1}\left(v k_{t}(t)\right)$,

$$
\tau_{K}\left(C_{t}\right)=4 \int_{[0, t]^{2}} \frac{C(x, y) \partial_{12}^{2} C(x, y)}{\partial_{1} C(x, t) \partial_{2} C(t, y)} \cdot \frac{\partial_{1} C(x, t) \partial_{2} C(t, y)}{C(t, t)^{2}} d x d y-1 .
$$

Analogously, 


$$
\begin{aligned}
\rho_{S}\left(C_{t}\right) & =12 \int_{[0,1]^{2}}\left(C_{t}(u, v)-u v\right) d u d v \\
& =12 \int_{[0, t]^{2}}\left(\frac{C(x, y)}{C(t, t)}-\frac{C(x, t) C(t, y)}{C(t, t)^{2}}\right) \frac{\partial_{1} C(x, t) \partial_{2} C(t, y)}{C(t, t)^{2}} d x d y,
\end{aligned}
$$

which concludes the proof.

Notice that the measures of association of $C_{t}$ only depend on the value of $C$ on the subdomain $[0, t]^{2}$.

Finally, together with $\tau_{K}$ and $\rho_{S}$, a measure of dependence may be considered for the process $\left(C_{t}\right)_{t \in(0,1]}$. Here we consider the Schweizer-Wolff's index $\sigma$ (see [13]), given by

$$
\sigma(C)=12 \int_{0}^{1} \int_{0}^{1}|C(u, v)-u v| d u d v .
$$

By the same arguments in the proof of Proposition 4, it straightly follows that

$\sigma\left(C_{t}\right)=\frac{12}{C(t, t)^{4}} \int_{0}^{t} \int_{0}^{t}|C(u, v) C(t, t)-C(u, t) C(t, v)| \partial_{1} C(u, t) \partial_{2} C(t, v) d u d v$.

It is immediate that, if $C$ is PQD, then $\sigma(C)=\rho_{S}(C)$. Therefore, when $C$ is hyper-PQD, $\sigma\left(C_{t}\right)=\rho_{S}\left(C_{t}\right)$. However, in general, $\sigma\left(C_{t}\right)$ is not directly obtained from $\rho_{S}\left(C_{t}\right)$ (see [11, Examples 5.18, 5.19]).

Example 2. Let us consider the copula

$$
C(u, v)=u v+u v(1-u)(1-v) .
$$

In view of Proposition 4, for every $t \in(0,1]$, we have

$$
\tau_{K}\left(C_{t}\right)=\frac{2 t^{2}}{9\left(2-2 t+t^{2}\right)^{2}} .
$$

In particular, $\tau_{K}\left(C_{t}\right) \rightarrow 0$ as $t \rightarrow 0^{+}$. Analogously, for every $t \in(0,1]$, we have

$$
\rho_{S}\left(C_{t}\right)=\frac{t^{6}}{3}
$$

and $\rho_{S}\left(C_{t}\right) \rightarrow 0$ as $t \rightarrow 0^{+}$. Intuitively, the residual lifetimes are asymptotically (as the time $\tau$ tends to infinity) uncorrelated.

A stronger conclusion can be achieved by means of the $\sigma$ index. Since $C$ is TP2 and hence hyper-PQD, $\sigma\left(C_{t}\right)=\rho_{S}\left(C_{t}\right)$ for any $t$. Thus we also have $\sigma\left(C_{t}\right) \rightarrow 0$ as $t \rightarrow 0^{+}$, implying that the residual lifetimes are asymptotically independent. 


\section{Conclusions}

We have considered a copula process that allows to study the dependence behaviour of a random vector of lifetimes $\mathbf{X}$, knowing that all the components are surviving up to time $\tau$. The study of such a copula process provides a way for looking at the tail dependence of the joint distribution of the vector. Moreover, the association measures related to the process may provide another way for expressing how the residual lifetimes evolve when the time increases.

Acknowledgements The first author acknowledges the support of Free University of Bozen-Bolzano, School of Economics and Management, via the project "Stochastic Models for Lifetimes".

\section{References}

1. Charpentier, A., Juri, A.: Limiting dependence structures for tail events, with applications to credit derivatives. J. Appl. Probab. 43(2), 563-586 (2006)

2. Cuadras, C.M., Augé, J.: A continuous general multivariate distribution and its properties. Comm. Statist. A-Theory Methods 10(4), 339-353 (1981)

3. Durante, F.: A new class of symmetric bivariate copulas. J. Nonparametr. Stat. 18(78), 499-510 (2006)

4. Durante, F., Foschi, R., Spizzichino, F.: Threshold copulas and positive dependence. Statist. Probab. Lett. 78(17), 2902-2909 (2008)

5. Durante, F., Jaworski, P.: Invariant dependence structure under univariate truncation. Statistics 46, 263-267 (2012)

6. Durante, F., Jaworski, P., Mesiar, R.: Invariant dependence structures and Archimedean copulas. Statist. Probab. Lett. 81(12), 1995-2003 (2011)

7. Durante, F., Kolesárová, A., Mesiar, R., Sempi, C.: Semilinear copulas. Fuzzy Sets and Systems 159(1), 63-76 (2008)

8. Foschi, R.: Semigroups of semi-copulas and a general approach to hyper-dependence properties (2012). Under revision.

9. Foschi, R., Spizzichino, F.: Semigroups of semicopulas and evolution of dependence at increase of age. Mathware \& Soft Computing XV(1), 95-111 (2008)

10. Jaworski, P., Durante, F., Härdle, W., Rychlik, T. (eds.): Copula Theory and its Applications, Lecture Notes in Statistics - Proceedings, vol. 198. Springer, Berlin Heidelberg (2010)

11. Nelsen, R.B.: An introduction to copulas, second edn. Springer Series in Statistics. Springer, New York (2006)

12. Schmid, F., Schmidt, R., Blumentritt, T., Gaisser, S., Ruppert, M.: Copula-based measures of multivariate association. In: P. Jaworski, F. Durante, W. Härdle, T. Rychlik (eds.) Copula Theory and its Applications, Lecture Notes in Statistics - Proceedings, vol. 198, pp. 209-236. Springer, Berlin Heidelberg (2010)

13. Schweizer, B., Wolff, E.F.: On nonparametric measures of dependence for random variables. Ann. Statist. 9(4), 879-885 (1981) 